\title{
Exploring the Differences between Hispanic Men and Women with Type 2 Diabetes Mellitus Using the Hispanic Community Health Study/Study of Latinos (HCHS/SOL)
}

\author{
Madeleine Morin, Erin St. Onge* \\ University of Florida College of Pharmacy, Orlando, FL, USA \\ Email: *stonge@cop.ufl.edu
}

How to cite this paper: Morin, M. and St. Onge, E. (2019) Exploring the Differences between Hispanic Men and Women with Type 2 Diabetes Mellitus Using the Hispanic Community Health Study/Study of Latinos (HCHS/SOL). Food and Nutrition Sciences, 10, 713-718.

https://doi.org/10.4236/fns.2019.107052

Received: May 22, 2019

Accepted: July 6, 2019

Published: July 9, 2019

Copyright $\odot 2019$ by author(s) and Scientific Research Publishing Inc. This work is licensed under the Creative Commons Attribution International License (CC BY 4.0).

http://creativecommons.org/licenses/by/4.0/

\begin{abstract}
Studies have shown Hispanic patients are at an increasing risk of developing type 2 diabetes; however, little is known about whether gender plays a role. Several studies were examined to help answer this question. Results of studies found female Hispanic patients tend to be more sedentary and thus struggle more with obesity, while male Hispanic patients tend to be more at risk due to smoking status and hypercholesterolemia. In addition, psychological distress and chronic stress were more likely to affect the risk for diabetes in men while immigration status was more likely to affect cardiovascular health in women. More studies are needed to fully understand the role of gender on diabetes risk; however, practitioners can utilize known information in order to tailor treatment to male or female Hispanic patients.
\end{abstract}

\section{Keywords}

Hispanic, Diabetes, Risk, Cardiovascular, Gender

\section{Introduction}

Previous research has well established that those of Hispanic and Latino ethnicity are at a higher risk of type 2 diabetes mellitus than non-Hispanic Caucasians [1]. However, to this date, there has been no research investigating the differences between genders within the Hispanic ethnicity. Although there are similar rates of diabetes in both men and women, after adjusting for factors such as age, BMI, Hispanic background, and years lived in the U.S. [2], this manuscript seeks to examine possible inter-gender differences in contributing factors to type 2 
diabetes mellitus.

\section{Methods}

Chosen articles were limited to those which gathered information using the Hispanic Community Health Study/Study of Latinos (HCHS/SOL). This was done in order to allow for a study pool which was consistent in demographics and protocol. The HCHS/SOL was a prospective cohort study of the Hispanic population conducted at multiple centers across the nation. It included 16,415 randomly selected participants from the Bronx, Chicago, Miami, and San Diego, known as field centers or sites. Several diverse backgrounds were represented, including those from South America, the Caribbean, and Central America. The goal was to evaluate disease states such as cardiovascular disease, pulmonary disease, and diabetes [3].

PubMed was searched using the phrase "HCHS/SOL and diabetes". Thirty-one articles appeared in the search results. The terms, "gender", "sex", "male", and "female" were searched for within each paper. If the authors stated they controlled for gender or sex in their study, adjusted their statistics using gender or sex, or did not report separate results for males and females, the study was excluded from the review. In addition, any articles unrelated to diabetes or possible risk factors for diabetes were excluded. Nine studies were ultimately included. Within the remaining nine studies, the terms "gender", "sex", "male", and "female" were again searched, and the author's results were interpreted and synthesized within the review.

\section{Results}

Results of the large HCHS/SOL study were examined by researchers from various aspects. These included analyzing factors which affect CV risk, evidence of disease control, psychological distress and chronic stress, as well as psychosocial support. The results of the nine studies included in this review are outlined in Table 1.

One study found Hispanic women with diabetes mellitus engage in more sedentary time than their male counterparts, after adjusting for age $(p=0.002)$ [4]. They were also less likely to engage in moderate to vigorous physical activity ( $\mathrm{p}$ $<0.001)$. Additionally, the results of this study found Hispanic patients with diabetes who decrease the time spent in a sedentary lifestyle, increase their chance of reaching both glycemic and triglyceride goals [4].

A survey conducted of the HCHS/SOL participants measured the prevalence of obesity, hypercholesterolemia, smoking, hypertension, and diabetes mellitus [5]. Participants were stratified into their respective backgrounds, including $\mathrm{Cu}$ ban, Dominican, Mexican, Puerto Rican, Central American, and South American. The results for all backgrounds were averaged together and included in the data under the category of "all". Women had a higher prevalence of obesity than men across all backgrounds. On average, the rates of obesity were $42.6 \%$ for 
Table 1. Results of Analyses of the HCHS/SOL Study.

\begin{tabular}{|c|c|c|}
\hline Reference & Sample Size & Sex Stratified Results \\
\hline Wang [4] & 1699 & $\begin{array}{l}\text { - Hispanic women spend more time sedentary compared to Hispanic } \\
\text { men } \\
\text { - Hispanic women are less likely to engage in moderate to vigorous } \\
\text { physical activity compared to Hispanic men } \\
\text { - Less time spent sedentary for both sexes improved CVD risk factor } \\
\text { control }\end{array}$ \\
\hline Daviglus [5] & 16,415 & $\begin{array}{l}\text { - Hispanic/Latino women have higher rates of obesity than } \\
\text { Hispanic/Latino men } \\
\text { - Hispanic/Latino men have higher rates of smoking and } \\
\text { hypercholesterolemia than Hispanic/Latino women } \\
\text { - Rates of diabetes and hypertension were similar among both sexes in } \\
\text { Hispanic/Latino subjects }\end{array}$ \\
\hline Greenblatt [6] & 15,945 & $\begin{array}{l}\text { - Hispanic women with diabetes had higher mean number of missing } \\
\text { teeth than Hispanic men } \\
\text { - Hispanic women enrolled in Medicaid had a higher mean number of } \\
\text { missing teeth than those with no or other insurance coverage } \\
\text { - Less Hispanic men were enrolled in Medicaid than Hispanic women }\end{array}$ \\
\hline Castañeda [7] & 15,781 & $\begin{array}{l}\text { - Psychological distress and chronic stress were associated with higher } \\
\text { rates of diabetes in Hispanic men than Hispanic women }\end{array}$ \\
\hline McCurley [8] & 3923 & $\begin{array}{l}\text { - Chronic stress was more strongly associated with higher fasting } \\
\text { glucose levels in Hispanic men than women } \\
\text { - Chronic stress resulted in no difference in postload glucose or Alc } \\
\text { between sexes }\end{array}$ \\
\hline Kershaw [9] & 15,047 & $\begin{array}{l}\text { - Length of residence in US significantly affected CV risk in Hispanic } \\
\text { women; this trend was not observed in Hispanic men }\end{array}$ \\
\hline Gallo [10] & 5181 & $\begin{array}{l}\text { - Higher functional and structural support was associated with lower } \\
\text { rates of diabetes in both Hispanic men and women }\end{array}$ \\
\hline
\end{tabular}

women and $36.5 \%$ for men. However, men had a higher prevalence of smoking and hypercholesterolemia. The rates of current smokers were $25.7 \%$ for men and $15.2 \%$ for women. Men had higher rates of smoking when compared to women of all backgrounds except Dominican. The overall average rate of hypercholesterolemia was $51.7 \%$ for men and $36.9 \%$ for women. Whether or not women or men had higher rates of diabetes and hypertension varied across their backgrounds, but all rates were very similar, differing only in a fraction of a percent between genders. The rates for diabetes and hypertension were very similar on average at $16.7 \%$ for men and $17.2 \%$ for women and $25.4 \%$ for men and $23.5 \%$ for women, respectively [5].

Diabetes is correlated with poor oral health; one study examined the relationship between diabetes and tooth loss among patients with diabetes in the HCHS/SOL. They found overall, women had a higher mean number of missing teeth compared to men. Additionally, women enrolled in Medicaid had a higher number of missing teeth on average, when compared to women who either had no or another form of health insurance. Men did not display this relationship, and fewer men were enrolled in Medicaid than women. The researchers acknowledged this relationship is unusual because previous studies established a 
lack of access to healthcare can increase the risk of poor oral health in patients with diabetes [6].

Psychological distress and chronic stress were more strongly associated with rates of diabetes for men than women. For example, in males, diabetes was strongly correlated with depression and anxiety after adjusting for age, background, education, income, health insurance, the language of preference, country of birth, and field center $(\mathrm{p}<0.05)$. This association was not observed in females [7]. In men, there was a stronger association between chronic stress and impaired fasting glucose levels $(\mathrm{p}<0.001)$ [8]. Chronic stress was measured using a self-reported scale which asked about life domains such as relationships and work. Glucose control was measured using fasting glucose, post-load glucose, and HbAlc. The researchers acknowledged that while there was effect modification by sex for fasting glucose $(\mathrm{p}<0.1)$, this was not present for post-load glucose or HbAlc $(p>0.05)$ [8]. More studies are needed to determine if chronic stress affects post-load glucose and $\mathrm{HbA1c}$ in men

Women who had recently immigrated to the United States within the past 10 years had a healthier cardiovascular (CV) profile than those who had been living in the United States for more than 10 years and those born in the United States [9]. Women living in the U.S. for less than 10 years had a low-CV risk prevalence of $15.4 \%$, compared to $9.4 \%$ for women living in the U.S. for 10 or more years and $9.2 \%$ for women born in the U.S. They were also two times more likely to have a better CV profile than women born in the U.S. This relationship was not observed in men. In fact, $4.5 \%$ of men living in the U.S. for less than 10 years had a favorable profile, while $6.0 \%$ of those born in the US had a favorable CV profile. Favorable CV health was defined as "untreated cholesterol levels less than $200 \mathrm{mg} / \mathrm{dL}$, untreated blood pressure less than $120 / 80 \mathrm{mmHg}$, BMI $<25.0$ $\mathrm{kg} / \mathrm{m}^{2}$, fasting plasma glucose $<100 \mathrm{mg} / \mathrm{dl}$ and no history of diabetes mellitus; not currently smoking; and no major ECG abnormalities." These results confirm previous theories that acculturation in the United States decreases CV health. Those living in the U.S. for less than 10 years are more likely to continue their native-born cultural practices when compared to those born in the U.S. or living in the U.S for more than 10 years. It is interesting to note this relationship was only confirmed in female participants of the HCHS/SOL [9].

Structural and functional support have been linked to a lower prevalence of diabetes, with no differences between the sexes [10]. Structural support is defined as "number and diversity of social roles or frequency of social contact that one experiences," and was measured by the number of "high contact social roles" the participant has. Functional support is defined as "the perception that supports resources, such as material aid, emotional support, companionship or information, would be available from one's social network, if needed," and was measured by the 12-item Interpersonal Support Evaluation List (ISEL-12). The researchers used an unadjusted, logistic regression model to investigate differences in sociodemographic covariates, and their results were consistent between genders (95\% CI $0.97[0.79,1.20])[10]$. 
Table 2. Predominant diabetes risk factors and comorbidities in hispanic patients.

\begin{tabular}{cc}
\hline Female & Male \\
\hline Sedentary lifestyle & Smoking \\
Obesity & Hypercholesterolemia \\
Poor dentition & Chronic stress \\
\hline
\end{tabular}

\section{Discussion}

Based on the studies evaluated, poor mental health was more strongly linked to diabetes prevalence and symptoms in Hispanic men than women. Prior research has found diabetes decreases one's quality of life, including one's cognitive health [11]. More research should be conducted in order to determine the effect of adding a mental health component to diabetes treatment for Hispanic men. For example, a mental health screening questionnaire could be included with each diabetes appointment.

Women displayed more obesity-related factors than men. More women engaged in sedentary time and had higher obesity rates than men. The Centers for Disease Control and Prevention found $87.5 \%$ of Hispanic patients with diabetes are also obese [12]. Diabetes treatment and prevention for Hispanic women should focus on this component. Practitioners should be aware Hispanic women may be more likely to struggle with obesity and should help enroll their patients in weight loss programs along with their diabetes treatments. They should also provide assistance in finding healthier, low-cost food options in the area.

Although Hispanic women might have higher rates of obesity than men, and psychological components are associated with diabetes rates among Hispanic men, this does not necessarily mean they should be treated completely differently. Practitioners should not dismiss the idea that Hispanic women might have poor mental health, for example. However, practitioners should be aware of these correlations to assist in a holistic treatment of their patient's diabetes. These gender differences (summarized in Table 2) should be explored to establish specialized prevention, early detection, and treatment programs.

\section{Conflicts of Interest}

The authors declare no conflicts of interest regarding the publication of this paper.

\section{References}

[1] Centers for Disease Control and Prevention. National Diabetes Fact Sheet: National Estimates and General Information on Diabetes and Prediabetes in the United States. https://www.cdc.gov/diabetes/pubs/pdf/ndfs_2011.pdf

[2] Schneiderman, N., Llabre, M., Cowie, C.C., et al. (2014) Prevalence of Diabetes among Hispanics/Latinos from Diverse Backgrounds: The Hispanic Community Health Study/Study of Latinos (HCHS/SOL). Diabetes Care, 37, 2233-2239. https://doi.org/10.2337/dc13-2939 
[3] National Institutes of Health. Hispanic Community Health Study/Study of Latinos Data Book: A Report to the Communities (NIH Publication No. 13-7951). https://www.nhlbi.nih.gov/files/docs/resources/NHLBI-HCHSSOL-English-508.pdf

[4] Wang, X., Strizich, G., Hua, S., et al. (2017) Objectively Measured Sedentary Time and Cardiovascular Risk Factor Control in US Hispanics/Latinos with Diabetes Mellitus: Results from the Hispanic Community Health Study/Study of Latinos (HCHS/SOL). Journal of the American Heart Association, 6, e004324. https://doi.org/10.1161/JAHA.116.004324

[5] Daviglus, M.L., Pirzada, A. and Talavera, G.A. (2014) Cardiovascular Disease Risk Factors in the Hispanic/Latino Population: Lessons from the Hispanic Community Health Study/Study of Latinos (HCHS/SOL). Progress in Cardiovascular Diseases, 57, 230-236. https://doi.org/10.1016/j.pcad.2014.07.006

[6] Greenblatt, A.P., Salazar, C.R., Northridge, M.E., et al. (2016) Association of Diabetes with Tooth Loss in Hispanic/Latino Adults: Findings from the Hispanic Community Health Study/Study of Latinos. BMJ Open Diabetes Research \& Care, 4 , e000211. https://doi.org/10.1136/bmjdrc-2016-000211

[7] Castañeda, S.F., Buelna, C., Giacinto, R.E., et al. (2016) Cardiovascular Disease Risk Factors and Psychological Distress among Hispanics/Latinos: The Hispanic Community Health Study/Study of Latinos (HCHS/SOL). Preventive Medicine, 87, 144-150. https://doi.org/10.1016/j.ypmed.2016.02.032

[8] McCurley, J.L., Mills, P.J., Roesch, S.C., et al. (2015) Chronic Stress, Inflammation, and Glucose Regulation in U.S. Hispanics from the HCHS/SOL Sociocultural Ancillary Study. Psychophysiology, 52, 1071-1079. https://doi.org/10.1111/psyp.12430

[9] Kershaw, K.N., Giacinto, R.E., Gonzalez, F., et al. (2016) Relationships of Nativity and Length of Residence in the U.S. with Favorable Cardiovascular Health among Hispanics/Latinos: The Hispanic Community Health Study/Study of Latinos (HCHS/SOL). Preventive Medicine, 89, 84-89. https://doi.org/10.1016/j.ypmed.2016.05.013

[10] Gallo, L.C., Fortmann, A.L., McCurley, J.L., et al. (2015) Associations of Structural and Functional Social Support with Diabetes Prevalence in U.S. Hispanics/Latinos: Results from the HCHS/SOL Sociocultural Ancillary Study. Journal of Behavioral Medicine, 38, 160-170. https://doi.org/10.1007/s10865-014-9588-Z

[11] Trikkalinou, A., Papazafiropoulou, A.K. and Melidonis, A. (2017) Type 2 Diabetes and Quality of Life. World Journal of Diabetes, 8, 120-129. https://doi.org/10.4239/wjd.v8.i4.120

[12] Centers for Disease Control and Prevention. National Diabetes Statistics Report, 2017.

https://www.cdc.gov/diabetes/pdfs/data/statistics/national-diabetes-statistics-report. pdf 